\title{
Review of Laboratory Tests on Athletes
}

\author{
Mahsa Amirhosseini' ${ }^{1 *}$ and Saeed Kamali ${ }^{2}$ \\ ${ }^{1} \mathrm{MA}$ in Sport Management, Farhangian University, Iran \\ ${ }^{2} \mathrm{PhD}$ in Sport Physiology, Batumi Shota Rustaveli University, Georgia \\ *Corresponding author: Mahsa Amirhosseini, Farhangian University, Gorgan, Iran
}

ARTICLE INFO
Received: 幽 November 13, 2020
Published:

Citation: Mahsa Amirhosseini, Saeed Kamali. Review of Laboratory Tests on Athletes. Biomed J Sci \& Tech Res 32(1)-2020. BJSTR. MS.ID.005192.

\section{ABSTRACT}

The general expression of experiments and their division and experiments before and after practice is not a scientific and correct work. First, the cases should be examined in detail. Here is a summary of how the experiments were selected, although there is a need for a lot of explanation and analysis. Routine tests are tests that are generally performed on most people and are called checksum. Health Control Tests The goal of exercise is to control blood factors and promote good health. For example, a person with high blood fats, a person with high blood fats, or people with high blood sugar go to the gym.

Keywords: Professional exercise; Medical test; Exercise test; Cardiorespiratory disease

\section{Mini Review}

Tests in professional athletes depending on the type of sport, these tests can be very varied and variable. For example, if the athlete is losing weight, but in wrestling and weightlifting, etc., specialized tests in addition to routine tests can be helpful. If the athlete does not gain proper weight despite good nutrition, specialized tests and cortisol, etc. [1] in certain seasons of the year, such as the hot season, part of the calcium, calcium, sodium, phosphorus can be helpful. These tests are very extensive in athletes and can hold multiple sessions depending on the discipline. There is also a need for basic information in the body for participants. For example, items such as premature fatigue, weight loss, weight gain, blood pressure, muscle analysis, and the like were discussed separately [2]. Medical evaluation is the first step in prescribing exercise and a complete medical evaluation, and it is appropriate for anyone who decides to participate in a regular exercise program to have a medical examination record [3]. This is especially true for people of different ages who have symptoms of coronary heart disease or who are 35 years of age or older but have no symptoms of coronary heart disease.

\section{Medical Examination}

A medical test is a medical procedure performed to detect, diagnose, or monitor diseases, disease processes, susceptibility, or to determine a course of treatment. Medical tests such as, physical and visual exams, diagnostic imaging, genetic testing, chemical and cellular analysis, relating to clinical chemistry and molecular diagnostics, are typically performed in a medical setting [4,5]. The medical test must be accurate and correct and include the following:

1. A questionnaire with a clear review of medical history should include personal and family history and current health habits such as smoking, diet, exercise, etc. Special emphasis is placed on any history of chest pain, irregular heartbeat or cardiovascular disease.

2. Physical examination, where special emphasis should be placed on the symptoms of heart and lung symptoms and other issues that are identified in physical examinations. Bone and joint tests should also be included

3. Electrocardiogram (EKG) at rest

4. Systolic and diastolic blood pressure at rest

5. Blood transfusions in which fasting blood glucose and cholesterol and triglycerides are recommended, although not necessary.

6. Incremental sports test using (EKG) warning unless otherwise medically performed.

Exercise pressure test can be done with a workhorse and a motorized treadmill. The equipment should always be started first with a small degree of pressure and then gradually increased in 
intensity until it finally reaches the maximum work capacity. The main purpose of a blood pressure test is to put pressure on the heart to see any evidence of myocardial infarction or restriction of blood flow to the myocardium (i.e., myocardium). Cardiovascular electrocardiographic (ECG) symptoms (e.g., blood pressure and heart rate) and physical symptoms (such as shortness of breath and chest pain) become apparent during the test. The purpose of the pressure test is to diagnose people with cardiovascular disease, especially coronary heart disease. People do not develop anemia of the heart muscle right at the maximum level of repair.

\section{Sports Test}

Of the many benefits of fitness testing, the most important is to establish the strengths and weaknesses of the athlete. This is done by comparing fitness test results to other athletes in the same training group, the same sport, or in a similar population group. By comparing results to successful athletes in your sport, you can see the areas which need improvement, and the training program can be modified accordingly [5]. This way valuable training time can be used more efficiently. However, beware that some athletes perform well in their sport despite their physical or physiological attributes, and it may not be advantageous to be like them.

The main objectives of the sports test include important and effective points, which are summarized below.

1. Creating and manifesting obvious and hidden symptoms of heart diseases

2. Evaluating the practical cardiovascular capacity, especially as a means of recognizing people for martyr work or sports programs

3. Determining the capacity of physical work per minute or practical capacity by METS method

4. Assessing the body's reactions to physical or preventive fitness programs

5. Using it as a basis for sports prescription

6. Assisting in selecting and evaluating appropriate treatment methods

\section{ISSN: 2574-1241}

DOI: $10.26717 /$ BJSTR.2020.32.005192

Mahsa Amirhosseini. Biomed J Sci \& Tech Res

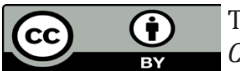

This work is licensed under Creative Commons Attribution 4.0 License

Submission Link: https://biomedres.us/submit-manuscript.php
7. Increasing the motivation of difference to enter and adhere to sports programs

\section{Conclusion}

Appropriate criteria set to determine who should receive a physician-supervised pressure test before starting an exercise program. The best advice is provided by doctors, but general recommendations are given, which are summarized below. Inactive men over the age of 35 and inactive postmenopausal women should have a pressure test before starting an exercise program. Anyone with chest pain or a history of heart disease, and anyone at any age who has significant risk factors for information on cardiovascular disease, should apply pressure before starting to practice the law and consider a medical evaluation program. These risk factors include a family history of cardiovascular disease, especially at a young age, a history of high blood pressure, a history of high cholesterol, especially low HDL, a history of diabetes, a smoker, especially if living a sedentary life with Other risk factors include having an abnormal ECG at rest.

\section{References}

1. Sundgot Borgen Jorunn (2013) How to minimise the health risks to athletes who compete in weight-sensitive sports review and position statement on behalf of the Ad Hoc Research Working Group on Body Composition, Health and Performance, under the auspices of the IOC Medical Commission. British journal of sports medicine 47(16): 10121022.

2. La Caille Lara J, Kim Nichols Dauner, Rachel J Krambeer, Jon Pedersen (2011) Psychosocial and environmental determinants of eating behaviors, physical activity, and weight change among college students: a qualitative analysis. Journal of American College Health 59(6): 531538.

3. Perri Michael G, Stephen D Anton, Patricia E Durning, Timothy U Ketterson, Sumner J Sydeman, et al. (2002) Adherence to exercise prescriptions: effects of prescribing moderate versus higher levels of intensity and frequency. Health Psychology 21(5): 452.

4. Sokolov Igor, V Kalaparthi, M Miljkovic, A Wang, JD Seigne, et al. (2018) Noninvasive diagnostic imaging using machine-learning analysis of nanoresolution images of cell surfaces: detection of bladder cancer Proceedings of the National Academy of Sciences 115(51): 1292012925.

5. Cronin John, Gord Sleivert (2005) Challenges in understanding the influence of maximal power training on improving athletic performance. Sports medicine 35(3): 213-234.

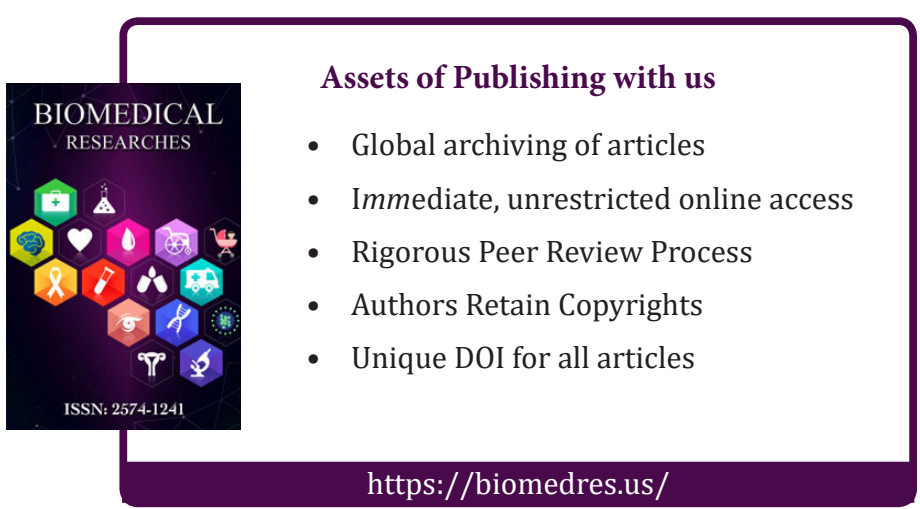

Copyright@ Mahsa Amirhosseini | Biomed J Sci \& Tech Res | BJSTR. MS.ID.005192. 